\title{
A simple evaluation procedure of the TAN calibration and the influence of non-ideal calibration elements on VNA S-parameter measurements
}

\author{
U. Stumper \\ Physikalisch-Technische Bundesanstalt, Bundesallee 100, 38116 Braunschweig, Germany
}

\begin{abstract}
For the 7-term general TAN (Through-AttenuatorNetwork) self-calibration method of a four-sampler vector network analyser (VNA), and for all derived calibration methods like TLN, TRL, TRM, TAR, or TMN, it is shown that a very simple evaluation procedure of the seven error terms is possible, even if the Through connection is replaced by a reflectionless network with known transmission. Expressions for the deviations of the measured S-parameters of two-port test objects (d.u.t.s) from the true values, which are caused by deviations of the modeled S-parameters of nonideal calibration elements ("standards") from their true values, are also presented. Additionally, it is shown that a TAN calibration is also possible in case of unequal reflections of the Network.
\end{abstract}

\section{Introduction}

The 7-term general TAN self-calibration procedure of a 4sampler VNA introduced by Eul and Schiek (1991), uses three two-ports as calibration elements, namely a reflectionless connection ("Through", T), a reflectionless two-port ("Attenuator", A) with unknown transmission coefficients, and a symmetrical two-port ("Network", N) with unknown S-parameters, but equal reflection coefficients at VNA test ports 1 and 2. From the uncorrected ("raw") reflection and transmission values determined for these three calibration elements, the seven error terms characterizing the VNA are calculated, and additionally, the five S-parameters of A and $\mathrm{N}$ are obtained.

The Attenuator A may be replaced by matched terminations (M) or by a matched precision line (L), and the Network $(\mathrm{N})$ by two equally reflecting terminations $(\mathrm{R})$, e.g. open circuits $(\mathrm{O})$. Thus, from the TAN

Correspondence to: U. Stumper

(ulrich.stumper@ptb.de) calibration other frequently used calibration procedures like TLN (Through-Line-Network), TRL (Through-ReflectLine), TRM (Through-Reflect-Match), TOM (ThroughOpen-Match), TAR (Through-Attenuator-Reflect), or TMN (Through-Match-Network) are derived.

The Through can also be replaced by a network where all four S-parameters are known. If instead of the Through connection (T) a network without reflection, but with known transmission parameters (including total transmission) is considered, a very simple evaluation procedure of the seven error terms is possible. This will be shown in Sect. 2 of this paper.

The error terms will deviate from their true values (as given by the VNA hardware), if modeling of the calibration elements $\mathrm{T}, \mathrm{A}$, and $\mathrm{N}$ is non-ideal, i.e. if their S-parameters deviate from the "ideal" values as assumed by the error correction software:

1. With the Through $(\mathrm{T})$, small reflection coefficients $\delta T_{11}, \delta T_{22}$, and small deviations $\delta T_{12}, \delta T_{21}$ of the transmission coefficients from their assumed values $T_{12}, T_{21}$ may occur, caused by transition resistance due to imperfect contacts, by cross-sectional discontinuities, or by small particles (e.g. lints) between the end planes of the connectors,

2. the Attenuator (A) may be of nonzero reflection $\delta M_{1}$, $\delta M_{2}$,

3. the Network (N) may be not perfectly symmetrical, e.g. may have unequal reflection coefficients $C+\delta C_{1}$, $C+\delta C_{2}$.

Consequently, with a non-ideal calibration, the measured Sparameters of a device under test (d.u.t.) will also deviate from their true values $S_{i k}$. In Sect. 3, these deviations $\delta S_{i k}$, assigned to the deviations from the true S-parameters associated with the calibration elements $\mathrm{T}, \mathrm{A}$, and $\mathrm{N}$, are derived 


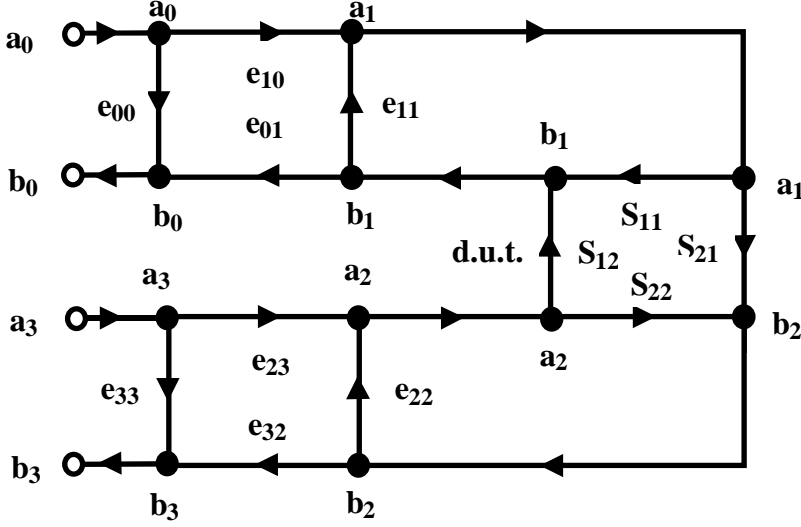

Fig. 1. Flow graph of 8-term error model. For a TAN calibration, the d.u.t. is replaced by the calibration elements: Through connection, Attenuator and Network. Signals $a_{1}, b_{1}$ refer to VNA test port 1 and $\mathrm{a}_{2}, \mathrm{~b}_{2}$ to test port 2 , respectively.

for the general TAN calibration procedure and for all calibration procedures derived from TAN. The influence of noise, non-linearity, and cross-talk effects on the raw values is not considered here.

\section{A simple calibration and measurement evaluation procedure}

The characteristics of a VNA are most commonly described in terms of cascaded matrices of the error terms (e.g. in Gronau, 2001). However, as shown here, the use of scattering matrices in the "linear-in-T" form depicted by Rytting (2001) allows, for the general TAN procedure, a direct derivation of the error terms themselves. This is different from the method described by Eul and Schiek (1991), where the S-parameters of Attenuator and Network are determined in a first step and are then used as "known" standard values in a subsequent calculation of the error terms.

\subsection{System equations}

Using the "linear-in-T" form of the VNA system equations, the S-parameters $S_{i k}$ of calibration elements or of a d.u.t. are linearly related to the $S$-parameters measured by the VNA (raw values $m_{i k}$ ) by 7 error terms $a, b, c, d, e, f$, and $g$ as

$a+S_{11} m_{11} b g-S_{11} c g+S_{21} m_{12} d=m_{11}$

$S_{12} m_{11} b g-S_{12} c g+S_{22} m_{12} d-m_{12} g=0$

$S_{11} m_{21} b g+S_{21} m_{22} d-S_{21} f=m_{21}$

$S_{12} m_{21} b g+e g+S_{22} m_{22} d-S_{22} f-m_{22} g=0$.

The $a, \ldots, g$ are reduced from 8 error terms $e_{i k}$ which are illustrated in Fig. 1. The reduction

$a=e_{00}, b=e_{11} e_{23} / e_{10}, c=\left(e_{00} e_{11}-e_{10} e_{01}\right) \cdot\left(e_{23} / e_{10}\right)$, $d=e_{22} \cdot e_{10} / e_{23}, e=e_{33}, f=\left(e_{22} e_{33}-e_{32} e_{23}\right)$.

$\left(e_{10} / e_{23}\right), g=e_{10} / e_{23}$

enables a simple calculation of $a, \ldots, g$ from the raw values and furthermore allows the determination of the deviations of $a, \ldots, g$.

\subsection{Calibration}

Ideally, the S-matrices of the Through connection (T), the Attenuator (A), and the Network (N) are

$\overline{\mathbf{T}}=\left(\begin{array}{cc}0 & T_{12} \\ T_{21} & 0\end{array}\right), \quad \overline{\mathbf{A}}=\left(\begin{array}{cc}0 & A \\ B & 0\end{array}\right)$, and $\overline{\mathbf{N}}=\left(\begin{array}{cc}C & D \\ E & C\end{array}\right)$

whereas the $T_{12}, T_{21}$ are known and $A, B, C, D$, and $E$ are unknown. The calibration elements $\mathrm{T}, \mathrm{A}$, and $\mathrm{N}$ may be nonreciprocal.

2.2.1 Determination of $a, b, c, d, e$, and $f$ using Through (T) and Attenuator (A)

Inserting the entries of $\overline{\mathbf{T}}$ and $\overline{\mathbf{A}}$ into the system Eqs. (1) to (4), for the Through (Index T) we obtain:

$a+T_{21} m_{12 \mathrm{~T}} d=m_{11 \mathrm{~T}}$

$T_{12} m_{11 \mathrm{~T}} b-T_{12} c-m_{12 \mathrm{~T}}=0$

$T_{21} m_{22 \mathrm{~T}} d-T_{21} f=m_{21 \mathrm{~T}}$

$T_{12} m_{21 \mathrm{~T}} b+e-m_{22 \mathrm{~T}}=0$

and for the Attenuator (Index A):

$a+B m_{12 \mathrm{~A}} d=m_{11 \mathrm{~A}}$

$A m_{11 \mathrm{~A}} b-A c-m_{12 \mathrm{~A}}=0$

$B m_{22 \mathrm{~A}} d-B f=m_{21 \mathrm{~A}}$

$A m_{21 \mathrm{~A}} b+e-m_{22 \mathrm{~A}}=0$.

To calculate the first six error terms $a, b, c, d, e$, and $f$, only the equation sets for these two calibration elements are necessary! In a first step $B$ is calculated from Eqs. (9) and (11). Using of Eqs. (5) and (7) we get:

$$
\begin{aligned}
& B=\left[\left(m_{11 \mathrm{~A}}-m_{11 \mathrm{~T}}\right)+T_{21} d \cdot m_{12 \mathrm{~T}}\right] /\left(d \cdot m_{12 \mathrm{~A}}\right) \\
& B=T_{21} m_{21 \mathrm{~A}} /\left[T_{21} d \cdot\left(m_{22 \mathrm{~A}}-m_{22 \mathrm{~T}}\right)+m_{21 \mathrm{~T}}\right] .
\end{aligned}
$$

Equating these expressions yields a quadratic equation which can be solved for $d$ :

$$
\begin{aligned}
& T_{21}^{2} d^{2} \cdot\left(m_{22 \mathrm{~A}}-m_{22 \mathrm{~T}}\right) \cdot m_{12 \mathrm{~T}}+\left(m_{11 \mathrm{~A}}-m_{11 T}\right) \cdot m_{21 T} \\
& +T_{21} d \cdot\left[\begin{array}{l}
\left(m_{11 \mathrm{~A}}-m_{11 \mathrm{~T}}\right) \cdot\left(m_{22 \mathrm{~A}}-m_{22 \mathrm{~T}}\right) \\
+m_{12 \mathrm{~T}} m_{21 \mathrm{~T}}-m_{21 \mathrm{~A}} m_{12 \mathrm{~A}}
\end{array}\right]=0 .
\end{aligned}
$$


Parameter $A$ is calculated from Eqs. (10) and (12). Using Eqs. (6) and (8) we get:

$$
\begin{aligned}
& A=T_{12} m_{12 \mathrm{~A}} /\left[T_{12} b \cdot\left(m_{11 \mathrm{~A}}-m_{11 \mathrm{~T}}\right)+m_{12 \mathrm{~T}}\right] \\
& A=\left[\left(m_{22 \mathrm{~A}}-m_{22 \mathrm{~T}}\right)+T_{12} b \cdot m_{21 \mathrm{~T}}\right] /\left(b \cdot m_{21 \mathrm{~A}}\right) .
\end{aligned}
$$

Equating these expressions again yields a quadratic equation which can be solved for $b$ :

$T_{12}^{2} b^{2} \cdot\left(m_{11 \mathrm{~A}}-m_{11 \mathrm{~T}}\right) \cdot m_{21 \mathrm{~T}}+\left(m_{22 \mathrm{~A}}-m_{22 \mathrm{~T}}\right) \cdot m_{12 \mathrm{~T}}$

$+T_{12} b \cdot\left[\begin{array}{l}\left(m_{11 \mathrm{~A}}-m_{11 \mathrm{~T}}\right) \cdot\left(m_{22 \mathrm{~A}}-m_{22 \mathrm{~T}}\right) \\ +m_{21 \mathrm{~T}} m_{12 \mathrm{~T}}-m_{12 \mathrm{~A}} m_{21 \mathrm{~A}}\end{array}\right]=0$.

With known $d$ and $b$, the parameters $a, c, e$, and $f$ are subsequently calculated using the Through Eqs. (5) to (8):

$a=\left(m_{11 \mathrm{~T}}-T_{21} m_{12 \mathrm{~T}} d\right)$

$c=\left(T_{12} m_{11 \mathrm{~T}} b-m_{12 \mathrm{~T}}\right) / T_{12}$

$e=\left(m_{22 \mathrm{~T}}-T_{12} m_{21 \mathrm{~T}} b\right)$

$f=\left(T_{21} m_{22 \mathrm{~T}} d-m_{21 \mathrm{~T}}\right) / T_{21}$.

\subsubsection{Transmission symmetry of Attenuator (A)}

In case of transmission symmetry of the Attenuator $(A=B)$, we equate Eqs. (13) and (17) and obtain

$\left(m_{11 \mathrm{~A}}-m_{11 \mathrm{~T}}\right) \cdot m_{21 \mathrm{~A}} b-\left(m_{22 \mathrm{~A}}-m_{22 \mathrm{~T}}\right) \cdot m_{12 \mathrm{~A}} d$

$-\left(T_{12} m_{12 \mathrm{~A}} m_{21 \mathrm{~T}}-T_{21} m_{21 \mathrm{~A}} m_{12 \mathrm{~T}}\right) \cdot b d=0$.

Equating Eqs. (14) and (16) yields

$$
\begin{aligned}
& T_{12} T_{21} \cdot\left(m_{11 \mathrm{~A}}-m_{11 \mathrm{~T}}\right) \cdot m_{21 \mathrm{~A}} b \\
& -T_{12} T_{21} \cdot\left(m_{22 \mathrm{~A}}-m_{22 \mathrm{~T}}\right) \cdot m_{12 \mathrm{~A}} d \\
& -\left(T_{12} m_{12 \mathrm{~A}} m_{21 \mathrm{~T}}-T_{21} m_{21 \mathrm{~A}} m_{12 \mathrm{~T}}\right)=0 .
\end{aligned}
$$

The subtraction of Eq. (24) from Eq. (23) yields:

$$
\begin{aligned}
& \left(1-T_{12} T_{21}\right) \cdot\left[\begin{array}{l}
\left(m_{11 \mathrm{~A}}-m_{11 \mathrm{~T}}\right) \cdot m_{21 \mathrm{~A}} b \\
-\left(m_{22 \mathrm{~A}}-m_{22 \mathrm{~T}}\right) \cdot m_{12 \mathrm{~A}} d
\end{array}\right] \\
& +(1-b d) \cdot\left(T_{12} m_{12 \mathrm{~A}} m_{21 \mathrm{~T}}-T_{21} m_{21 \mathrm{~A}} m_{12 \mathrm{~T}}\right)=0 .
\end{aligned}
$$

Since both Eqs. (23) and (24) describe linear relations between $b$ and $d$, only one of the quadratic Eqs. (15) or (18) has to be solved.

For a direct Through connection $\left(T_{12}=T_{21}=1\right)$, Eq. (25) becomes

$$
(1-b d) \cdot\left(m_{12 \mathrm{~T}} m_{21 \mathrm{~A}}-m_{21 \mathrm{~T}} m_{12 \mathrm{~A}}\right)=0 .
$$

The error terms $|b|$ and $|d|$ are small compared to 1 , viz $b d \neq 1$ :

$m_{12 \mathrm{~T}} m_{21 \mathrm{~A}}-m_{21 \mathrm{~T}} m_{12 \mathrm{~A}}=0$.

This relation between the raw values of Through and Attenuator is equivalent to the relation (33) of Engen (1979). Moreover, from Eq. (23) or (24) we obtain

$b=\frac{\left(m_{22 \mathrm{~A}}-m_{22 \mathrm{~T}}\right) \cdot m_{12 \mathrm{~A}}}{\left(m_{11 \mathrm{~A}}-m_{11 \mathrm{~T}}\right) \cdot m_{21 \mathrm{~A}}} \cdot d$.

\subsubsection{Determination of $g$}

To obtain the remaining error term $g$, we insert the entries of the Network matrix $\overline{\mathbf{N}}$ (Index N) into Eqs. (1) to (4):

$a+C m_{11 \mathrm{~N}} b g-C c g+E m_{12 \mathrm{~N}} d=m_{11 \mathrm{~N}}$

$D m_{11 \mathrm{~N}} b g-D c g+C m_{12 \mathrm{~N}} d-m_{12 \mathrm{~N}} g=0$

$C m_{21 \mathrm{~N}} b g+E m_{22 \mathrm{~N}} d-E f=m_{21 \mathrm{~N}}$

$D m_{21 \mathrm{~N}} b g+e g+C m_{22 \mathrm{~N}} d-C f-m_{22 \mathrm{~N}} g=0$.

First, the Network parameters $D$ and $E$ are eliminated. For $D$, from Eqs. (30) and (32), respectively, we obtain:

$D=m_{12 \mathrm{~N}} \cdot(g-C d) /\left(m_{11 \mathrm{~N}} b g-c g\right)$

$D=\left[\left(m_{22 \mathrm{~N}}-e\right) \cdot g-C \cdot\left(m_{22 \mathrm{~N}} d-f\right)\right] /\left(m_{21 \mathrm{~N}} b g\right)$.

Equating these expressions yields

$C=\frac{\left[\left(m_{11 \mathrm{~N}} b-c\right) \cdot\left(m_{22 \mathrm{~N}}-e\right)-m_{12 \mathrm{~N}} m_{21 \mathrm{~N}} b\right] \cdot g}{\left[\left(m_{11 \mathrm{~N}} b-c\right) \cdot\left(m_{22 \mathrm{~N}} d-f\right)-m_{12 \mathrm{~N}} m_{21 \mathrm{~N}} b d\right]}$.

For $E$, from Eqs. (29) and (31), respectively, we obtain:

$E=\left[\left(m_{11 \mathrm{~N}}-a\right)-C g \cdot\left(m_{11 \mathrm{~N}} b-c\right)\right] /\left(m_{12 \mathrm{~N}} d\right)$

$E=\left(m_{21 \mathrm{~N}}-C m_{21 \mathrm{~N}} b g\right) /\left(m_{22 \mathrm{~N}} d-f\right)$.

Equating these expressions yields a second equation for $C$ :

$C=\frac{\left[\left(m_{11 \mathrm{~N}}-a\right) \cdot\left(m_{22 \mathrm{~N}} d-f\right)-m_{12 \mathrm{~N}} m_{21 \mathrm{~N}} d\right]}{\left[\left(m_{11 \mathrm{~N}} b-c\right) \cdot\left(m_{22 \mathrm{~N}} d-f\right)-m_{12 \mathrm{~N}} m_{21 \mathrm{~N}} b d\right] \cdot g}$

Eliminating $C$ by equating Eqs. (35) and (38) gives

$g^{2}=\frac{\left[\left(m_{11 \mathrm{~N}}-a\right) \cdot\left(m_{22 \mathrm{~N}} d-f\right)-m_{12 \mathrm{~N}} m_{21 \mathrm{~N}} d\right]}{\left[\left(m_{11 \mathrm{~N}} b-c\right) \cdot\left(m_{22 \mathrm{~N}}-e\right)-m_{12 \mathrm{~N}} m_{21 \mathrm{~N}} b\right]}$

where $a, b, c, d, e$, and $f$ are already known. The terms $A$, $B, D, E$, and $C$ are not further used.

\subsubsection{Transmission symmetry of Network (N)}

In case of transmission symmetry of the Network $(\mathrm{N})(D=$ $E)$, we can formulate a second relation between the Through (T) and Network (N) raw values. Inserting Eq. (35) into Eqs. (33) or (34) yields

$$
D=\frac{(d e-f) \cdot m_{12 \mathrm{~N}}}{\left[\left(m_{11 \mathrm{~N}} b-c\right) \cdot\left(m_{22 \mathrm{~N}} d-f\right)-m_{12 \mathrm{~N}} m_{21 \mathrm{~N}} b d\right]},
$$

and inserting Eq. (38) into Eqs. (36) or (37)

$$
E=\frac{(a b-c) \cdot m_{21 \mathrm{~N}}}{\left[\left(m_{11 \mathrm{~N}} b-c\right) \cdot\left(m_{22 \mathrm{~N}} d-f\right)-m_{12 \mathrm{~N}} m_{21 \mathrm{~N}} b d\right]} .
$$

With $D=E$ :

$(d e-f) \cdot m_{12 \mathrm{~N}}=(a b-c) \cdot m_{21 \mathrm{~N}}$. 


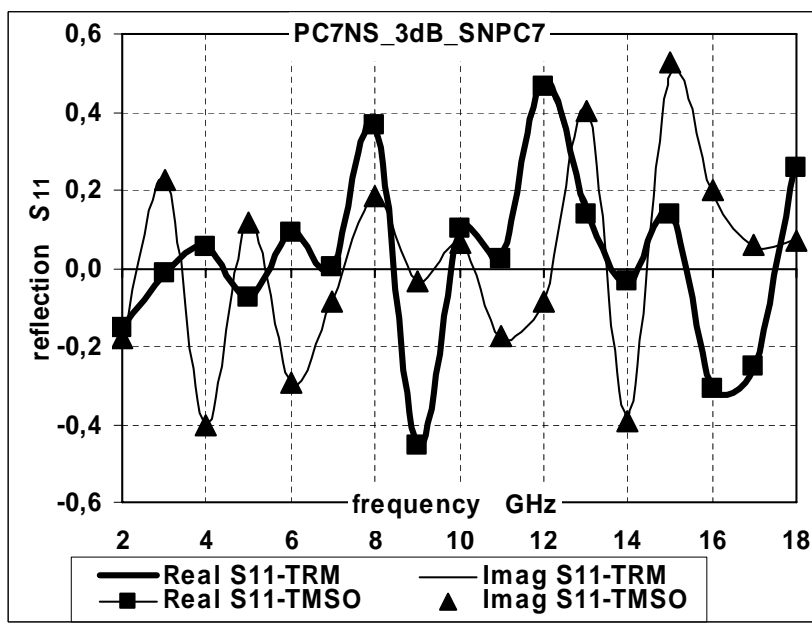

Fig. 2. Measured reflection coefficient $S_{11}$ of a high-reflective $3 \mathrm{~dB}$ attenuator using TRM calibration and TMSO calibration.

This relation is even valid if the reflection coefficients of the Network (N) are not equal.

Inserting Eqs. (19) to (22) into Eq. (42) yields:

$$
\left(T_{12} T_{21} b d-1\right) \cdot\left(T_{21} m_{21 \mathrm{~N}} m_{12 \mathrm{~T}}-T_{12} m_{12 \mathrm{~N}} m_{21 \mathrm{~T}}\right)=0 .
$$

For a direct Through connection $\left(T_{12}=T_{21}=1\right)$ we have

$(b d-1) \cdot\left(m_{21 \mathrm{~N}} m_{12 \mathrm{~T}}-m_{12 \mathrm{~N}} m_{21 \mathrm{~T}}\right)=0$,

and, since $b d \neq 1$ :

$m_{21 \mathrm{~N}} m_{12 \mathrm{~T}}-m_{12 \mathrm{~N}} m_{21 \mathrm{~T}}=0$

has to be fulfilled, similar to the relation (27), but here between the raw values of Through and Network.

\subsubsection{Calibration methods derived from TAN}

For the Through-Line-Network (TLN) calibration method, $A=B=L=\exp (-\gamma \cdot l)$, where $l$ and $\gamma$ are length and propagation coefficient of the transmission line.

For the Through-Attenuator-Reflect (TAR) calibration method, we get $D=E=0$. In this case, $m_{12 \mathrm{~N}}=m_{21 \mathrm{~N}}=0$ is valid in Eq. (39).

For the Line-Reflect-Line (LRL) calibration method, $T_{12}=T_{21}=L_{1}=\exp \left(-\gamma \cdot l_{1}\right), A=B=L_{2}=\exp \left(-\gamma \cdot l_{2}\right)$ and $D=E=0$ is fulfilled.

For the Through-Reflect-Line (TRL) calibration method, $T_{12}=T_{21}=1, A=B=L=\exp (-\gamma \cdot l)$ and $D=E=0$.

For the Through-Match-Network (TMN) calibration method, $A=B=0$. Hence, a direct determination of $b$ and $d$ from Eqs. (5) to (9) and Eq. (12) is possible.

For the Through-Reflect-Match (TRM) calibration method, $A=B=0$ and $D=E=0$.

\subsection{Evaluation of measurements}

With known error terms, the S-parameters $S_{i k}$ of a d.u.t. are obtained using the equations

$$
\begin{aligned}
& S_{11}=\left[\left(m_{11}-a\right) \cdot\left(m_{22} d-f\right)-m_{12} m_{21} d\right] /(M \cdot g) \\
& S_{12}=m_{12} \cdot(d e-f) / M \\
& S_{21}=m_{21} \cdot(a b-c) / M \\
& S_{22}=\left[\left(m_{11} b-c\right) \cdot\left(m_{22}-e\right)-m_{21} m_{12} b\right] \cdot g / M \\
& M=\left(m_{11} b-c\right) \cdot\left(m_{22} d-f\right)-m_{12} m_{21} b d .
\end{aligned}
$$

(cf. Rytting, 2001; Stumper, 2005c), where the $m_{i k}$ are now the raw values of the d.u.t. We recognize that for transmission measurements, only the error terms $a, \ldots, f$ and the calibration steps using Through and Attenuator are needed, while $g$ - and the calibration step using the Network - are only relevant for reflection measurements. The correct sign of $g$ is found by measuring a d.u.t. where the value of its reflection coefficient is approximately known (e.g. a short or open circuit).

\subsection{Verification}

To verify the new procedure, measurements have been performed, either using a TRM calibration method (derived from TAN with $A=B=D=E=0$ ) where $T_{12}=T_{21}=1$, or the well-known TMSO (or SOLT) calibration method. With the latter method, a 10-term VNA model is considered, which uses - in addition to the Through $(\mathrm{T})$ - well-defined matched terminations $(\mathrm{M})$, short-circuits $(\mathrm{S})$, and open-circuits $(\mathrm{O})$ as calibration standards.

To compare the measurements carried out with both calibration methods, a 8510B-type VNA, a single calibration kit including a broadband match (M), and the same d.u.t.s. were used. The raw values for TRM calibration and subsequent measurements were calculated according to Schiek (1999) from the four sampler signals provided by the VNA for both signal directions. For the TMSO calibration and subsequent measurements, the internal VNA firmware was used.

The d.u.t.s. were a set of high-reflecting $7 \mathrm{~mm}$ coaxial twoport devices with PC-7 connectors including attenuators of (nominal) attenuation $3 \mathrm{~dB}$ to $60 \mathrm{~dB}$ which were sandwiched between the side arms of two T-junctions with the feeding arms shortened (cf. Stumper, 2005b). Real and imaginary parts of the S-parameters of these devices varied between approximately -0.5 and +0.5 in the frequency range 2-18 GHz. A second d.u.t. set included low-reflective PC-7 attenuator pads of (nominal) attenuation $10 \mathrm{~dB}, 20 \mathrm{~dB}, 40 \mathrm{~dB}$, and $70 \mathrm{~dB}$. Examples of results of the comparison are shown in Fig. 2 to Fig. 5. Considering the moderate reflection (max. 0.06 at $18 \mathrm{GHz}$ ) of the broadband Match, we observed a good coincidence of measurements carried out with the two calibrations. 


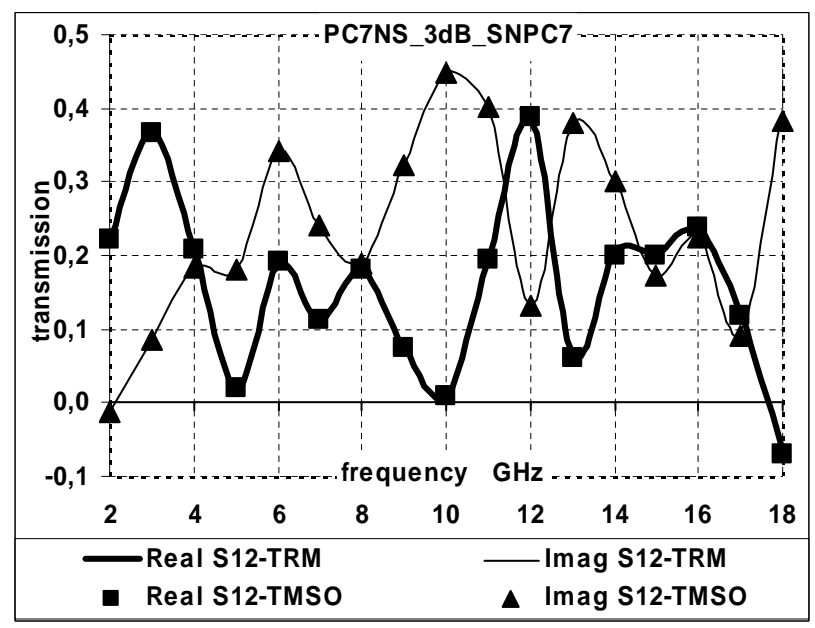

Fig. 3. Measured transmission coefficient $S_{12}$ of a high-reflective $3 \mathrm{~dB}$ attenuator using TRM calibration and TMSO calibration.

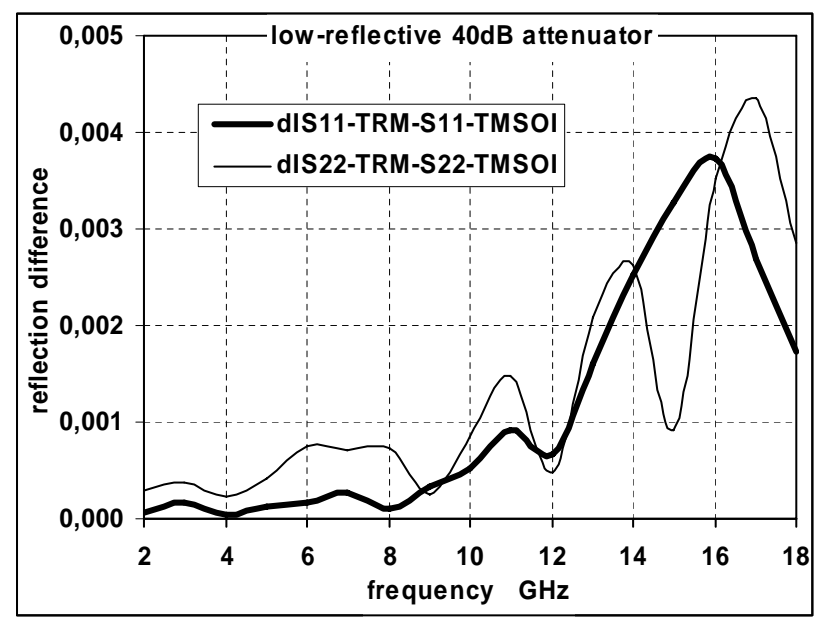

Fig. 4. Measured magnitude of vector difference of reflection coefficient $S_{11}$ (and of $S_{22}$ as well) of a low-reflective $40 \mathrm{~dB}$ attenuator using TRM calibration and TMSO calibration.

\section{Derivation of sensitivity coefficients for the TAN cali- bration}

\subsection{Calculation of the $\delta S_{i k}$}

In a first step, the deviations $\delta S_{i k}$ of a d.u.t. as functions of the deviations $\delta a, \delta b, \delta c, \delta d, \delta e, \delta f$, and $\delta g$ are calculated. The procedure has already been described by Stumper $(2005 \mathrm{~b}, \mathrm{c})$.

\subsection{Calculation of the deviation of error terms}

In a second step, the deviations $\delta a, \ldots, \delta g$ are calculated as functions of the deviations from the true S-parameters which are associated with the non-ideal calibration elements. The

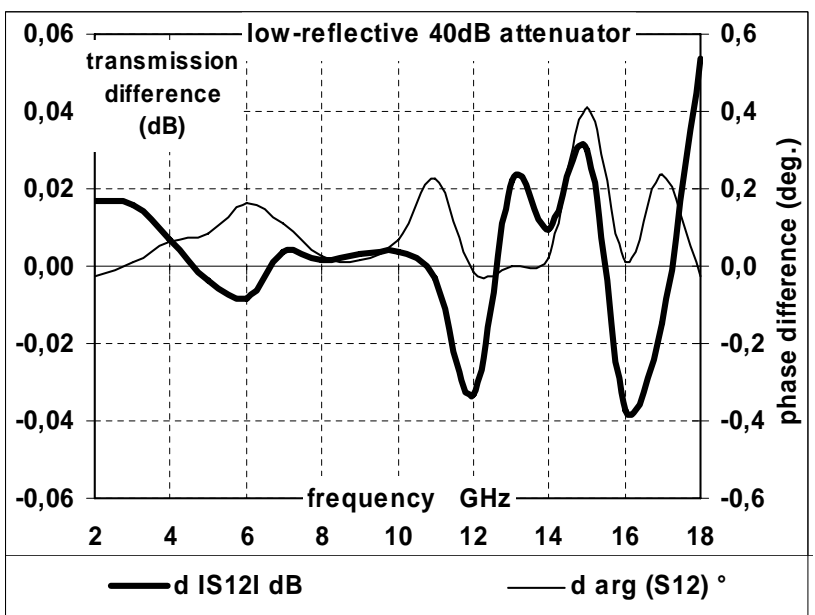

Fig. 5. Difference of transmission coefficient $S_{12}$ in dB, and transmission phase difference $\Delta \arg \left(S_{12}\right)$ in degree, of a low-reflective $40 \mathrm{~dB}$ attenuator, as measured using TRM calibration and TMSO calibration.

disturbed S-matrices of the non-ideal Through (T), Attenuator (A), and Network (N) are

$$
\begin{array}{ll}
\overline{\mathbf{T}}_{\mathrm{d}}=\left(\begin{array}{cc}
\delta T_{11} & T_{12}+\delta T_{12} \\
T_{21}+\delta T_{21} & \delta T_{22}
\end{array}\right), \quad \overline{\mathbf{A}}_{\mathrm{d}}=\left(\begin{array}{cc}
\delta M_{1} & A \\
B & \delta M_{2}
\end{array}\right), \\
\text { and } \overline{\mathbf{N}}_{\mathrm{d}}=\left(\begin{array}{cc}
C+\delta C_{1} & D \\
E & C+\delta C_{2}
\end{array}\right) .
\end{array}
$$

At first, we calculate disturbed raw values $m_{i k}+\delta m_{i k}$ by inserting the entries of the disturbed $\overline{\mathbf{T}}_{\mathrm{d}}, \overline{\mathbf{A}}_{\mathrm{d}}$, or $\overline{\mathbf{N}}_{\mathrm{d}}$, respectively, into the Eqs. (51) to (55) (Rytting, 2001; Stumper, 2005c) for $S_{11}, S_{12}, S_{21}$, and $S_{22}$, respectively:

$$
\begin{aligned}
& m_{11}=\left[\left(a-S_{11} c g\right) \cdot\left(g-S_{22} d\right)-S_{12} S_{21} c d g\right] / Q \\
& m_{12}=S_{12} \cdot(a b-c) \cdot g / Q \\
& m_{21}=S_{21} \cdot(d e-f) \cdot g / Q \\
& m_{22}=\left[\left(1-S_{11} b g\right) \cdot\left(e g-S_{22} f\right)-S_{12} S_{21} b f g\right] / Q \\
& Q=\left(1-S_{11} b g\right) \cdot\left(g-S_{22} d\right)-S_{12} S_{21} b d g .
\end{aligned}
$$

Next, the disturbed values $m_{i k}+\delta m_{i k}$ are inserted into the expressions (15), (18) to (22), and (39) for $a, \ldots, g$ to obtain the deviations $\delta a, \ldots, \delta g$.

As an example, the derivation of the deviation $\delta g_{\mathrm{N}}$ in case of a non-ideal Network $(\mathrm{N})$ is shortly outlined here. The terms $a, b, c, d, e$, and $f$ are neither depending on the Network parameters $D, E$, nor on the raw values $m_{i k \mathrm{~N}}$. Inserting the entries of $\overline{\mathbf{N}}_{\mathrm{d}}$ in Eq. (51), we obtain

$$
\begin{aligned}
& m_{11 \mathrm{~N}}+\delta m_{11 \mathrm{~N}}= \\
& \frac{\left[a-\left(C+\delta C_{1}\right) \cdot c g\right] \cdot\left[g-\left(C+\delta C_{2}\right) \cdot d\right]-D E c d g}{\left[1-\left(C+\delta C_{1}\right) \cdot b g\right] \cdot\left[g-\left(C+\delta C_{2}\right) \cdot d\right]-D E b d g},
\end{aligned}
$$




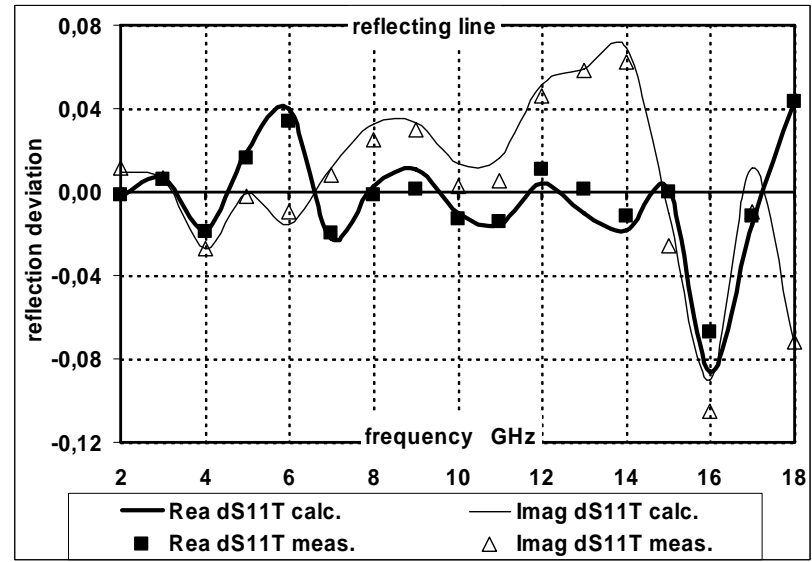

Fig. 6. Comparison of calculated and measured deviations $\delta S_{11 \mathrm{~T}}$ for TRM calibration and a high-reflective short line, using a nonideal Through (copper foil at the inner conductors at the VNA test ports), instead of an ideal Through.

and finally we get

$\delta m_{11 \mathrm{~N}}=\frac{(a b-c) \cdot g \cdot\left[(g-C d)^{2} \cdot \delta C_{1}+D E d^{2} \cdot \delta C_{2}\right]}{[(1-C b g) \cdot(g-C d)-D E b d g]^{2}}$.

For $\delta m_{12 \mathrm{~N}}, \delta m_{21 \mathrm{~N}}$, and $\delta m_{22 \mathrm{~N}}$ we obtain similar expressions.

From Eq. (39) we then establish

$\left(g+\delta g_{\mathrm{N}}\right)^{2}=\frac{\left\{\begin{array}{c}{\left[\left(m_{11 \mathrm{~N}}+\delta m_{11 \mathrm{~N}}\right)-a\right] \cdot\left[\left(m_{22 \mathrm{~N}}+\delta m_{22 \mathrm{~N}}\right) \cdot d-f\right]} \\ -\left(m_{12 \mathrm{~N}}+\delta m_{12 \mathrm{~N}}\right) \cdot\left(m_{21 \mathrm{~N}}+\delta m_{21 \mathrm{~N}}\right) \cdot d\end{array}\right\}}{\left\{\begin{array}{c}{\left[\left(m_{22 \mathrm{~N}}+\delta m_{22 \mathrm{~N}}\right)-e\right] \cdot\left[\left(m_{11 \mathrm{~N}}+\delta m_{11 \mathrm{~N}}\right) \cdot b-c\right]} \\ -\left(m_{12 \mathrm{~N}}+\delta m_{12 \mathrm{~N}}\right) \cdot\left(m_{21 \mathrm{~N}}+\delta m_{21 \mathrm{~N}}\right) \cdot b\end{array}\right\}}$.

Inserting the expressions obtained for $\delta m_{11 \mathrm{~N}}, \delta m_{12 \mathrm{~N}}, \delta m_{21 \mathrm{~N}}$, and $\delta m_{22 \mathrm{~N}}$ into Eq. (58), we finally obtain:

$\delta g_{\mathrm{N}}=\frac{g}{2 \cdot C} \cdot\left(\delta C_{1}-\delta C_{2}\right)$.

\subsection{Resulting sensitivity coefficients}

Inserting the results of the second step (Sect. 3.2) into Eqs. (32) to (35) as given in Stumper (2005c) (for $r_{i k}=S_{i k}$ there), we obtain sensitivity coefficients for the four Sparameters of a d.u.t. which are separately given for the deviations associated with the non-ideal Through (Index T), Attenuator (A), and Network (N). The expressions are not dependent on the error terms (i.e. not dependent on the VNA hardware):

$\delta S_{11 \mathrm{~T}}^{\mathrm{TAN}}=\left\{\begin{array}{l}\frac{\left(A B-S_{12} S_{21}\right)}{\left(T_{12} T_{21}-A B\right)} \cdot \delta T_{11} \\ -\frac{S_{11}^{2}}{\left(T_{12} T_{21}-A B\right)} \cdot \delta T_{22} \\ -\frac{S_{11} \cdot\left(A B+C^{2}-D E\right)}{2 \cdot C \cdot\left(T_{12} T_{21}-A B\right)} \cdot\left(\delta T_{11}-\delta T_{22}\right) \\ -\frac{S_{11}}{2} \cdot\left(\frac{\delta T_{12}}{T_{12}}+\frac{\delta T_{21}}{T_{21}}\right)\end{array}\right\}$

$\frac{\delta S_{12 \mathrm{~T}}^{\mathrm{TAN}}}{S_{12}}=\left\{\begin{array}{l}-\frac{S_{22}}{\left(T_{12} T_{21}-A B\right)} \cdot \delta T_{11}-\frac{\delta T_{12}}{T_{12}} \\ -\frac{S_{11}}{\left(T_{12} T_{21}-A B\right)} \cdot \delta T_{22}\end{array}\right\}$

$$
\begin{aligned}
& \delta S_{11 \mathrm{~A}}^{\mathrm{TAN}}=\left\{\begin{array}{l}
-\frac{\left(T_{12} T_{21}-S_{12} S_{21}\right)}{\left(T_{12} T_{21}-A B\right)} \cdot \delta M_{1} \\
+\frac{S_{11}^{2}}{\left(T_{12} T_{21}-A B\right)} \cdot \delta M_{2} \\
+\frac{S_{11} \cdot\left(T_{12} T_{21}+C^{2}-D E\right)}{2 \cdot C \cdot\left(T_{12} T_{21}-A B\right)} \cdot\left(\delta M_{1}-\delta M_{2}\right)
\end{array}\right\} \\
& \frac{\delta S_{12 \mathrm{~A}}^{\mathrm{TAN}}}{S_{12}}=\frac{\delta S_{21 \mathrm{~A}}^{\mathrm{TAN}}}{S_{21}}= \\
& \frac{S_{22}}{\left(T_{12} T_{21}-A B\right)} \cdot \delta M_{1}+\frac{S_{11}}{\left(T_{12} T_{21}-A B\right)} \cdot \delta M_{2} .
\end{aligned}
$$

To obtain the expressions for $\delta S_{21}$ and $\delta S_{22}$, index 1 has to be replaced by 2 and vice versa in these equations.

Nonequal reflection of the Network $(\mathrm{N})$ influences only the error term $g$ and, consequently, influences only the reflection coefficients of the d.u.t.:

$$
\begin{aligned}
& \delta S_{11 \mathrm{~N}}^{\mathrm{TAN}}=\frac{S_{11}}{2 \cdot C} \cdot\left(\delta C_{2}-\delta C_{1}\right) \\
& \delta S_{22 \mathrm{~N}}^{\mathrm{TAN}}=\frac{S_{22}}{2 \cdot C} \cdot\left(\delta C_{1}-\delta C_{2}\right) .
\end{aligned}
$$

There is no influence on transmission, viz $\delta S_{12 \mathrm{~N}}^{\mathrm{TAN}}=\delta S_{21 \mathrm{~N}}^{\mathrm{TAN}}=0$.

These expressions for the non-ideal Network remain the same for all calibration methods derived from TAN.

For the Line-Reflect-Line (LRL) calibration method we obtain the same sensitivity coefficients as given by Stumper (2005c) for small $e_{11}$. For the Through-Reflect-Line (TRL) calibration method we obtain the same sensitivity coefficients as given by Stumper (2005a).

For the Through-Reflect-Match (TRM) calibration method where $A=B=D=E=0$, and a Through (T) of total transmission $\left(T_{12}=T_{21}=1\right)$, we obtain the simple expressions:

$\delta S_{11 \mathrm{~T}}^{\mathrm{TRM}}=\left\{\begin{array}{l}-S_{12} S_{21} \cdot \delta T_{11}-S_{11}^{2} \cdot \delta T_{22} \\ -\frac{S_{11} \cdot C}{2} \cdot\left(\delta T_{11}-\delta T_{22}\right) \\ -\frac{S_{11}}{2} \cdot\left(\delta T_{12}+\delta T_{21}\right)\end{array}\right\}$

$\frac{\delta S_{12 \mathrm{~T}}^{\mathrm{TRM}}}{S_{12}}=-S_{22} \cdot \delta T_{11}-\delta T_{12}-S_{11} \cdot \delta T_{22}$

$\delta S_{11 \mathrm{~A}}^{\mathrm{TRM}}=\left\{\begin{array}{l}-\left(1-S_{12} S_{21}\right) \cdot \delta M_{1}+S_{11}^{2} \cdot \delta M_{2} \\ +\frac{S_{11} \cdot\left(1+C^{2}\right)}{2 \cdot C} \cdot\left(\delta M_{1}-\delta M_{2}\right)\end{array}\right\}$

$\frac{\delta S_{12 \mathrm{~A}}^{\mathrm{TRM}}}{S_{12}}=\frac{\delta S_{21 \mathrm{~A}}^{\mathrm{TRM}}}{S_{21}}=S_{22} \cdot \delta M_{1}+S_{11} \cdot \delta M_{2}$.

To obtain the expressions for $\delta S_{21}$ and $\delta S_{22}$, index 1 has to be replaced by 2 and vice versa in these equations.

\subsection{Experimental results}

For the TRL calibration method, Eqs. (60) to (65) have already been verified in the frequency range $2-18 \mathrm{GHz}$ (see Stumper, 2005a, b, c) showing good agreement between theory and experiment. 


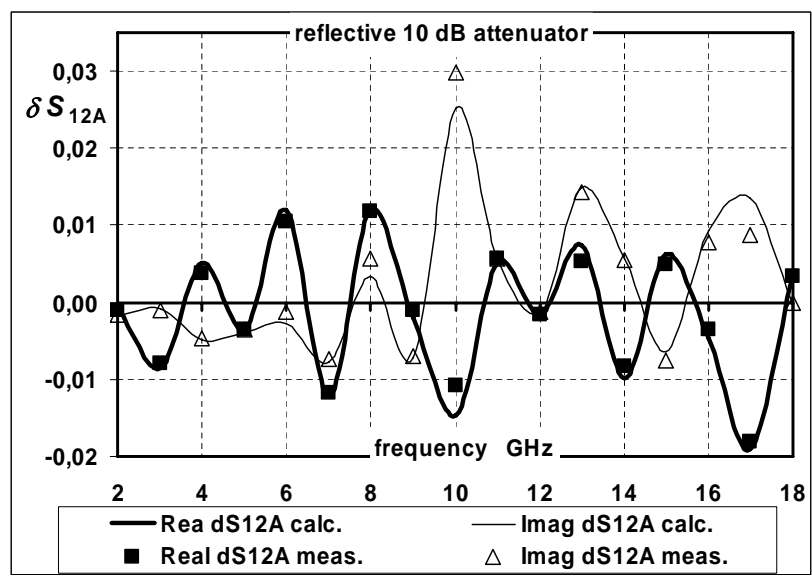

Fig. 7. Comparison of calculated and measured deviations $\delta S_{12} \mathrm{~A}$ for TRM calibration and a high-reflective $10 \mathrm{~dB}$ attenuator, using a mismatch of reflection 0.2 at the VNA test port 2, instead of an ideal Match.

For the TRM calibration method, Eqs. (64) to (69) have also been experimentally verified by use of the same set of coaxial (PC-7) high- and low-reflective two-ports as described by Stumper (2003a, 2005a, b, c). Only one of the elements Through, Reflect, and Match at a time was considered non-ideal. Examples are shown in Fig. 6 and Fig. 7. The agreement of theory and experiment is also good, with exception of the Match in the case of high-reflective d.u.t.s where some differences between calculated and measured deviations were observed.

\subsection{Comparison of TRL, TRM, and TMSO methods}

It is interesting to compare the influence of non-ideal calibration elements used for different calibration methods including the TMSO (or SOLT) calibration method. Some results are given here:

For TRM and for TMSO (Stumper, 2003a), a non-ideal Match has nearly the same influence on the deviations of the S-parameters for both high- and low-reflective d.u.t.s.

The influence of a non-ideal Through is different for TRL, TRM, and TMSO, and depends on the reflection of the d.u.t. In Fig. 8, the large influence of a non-ideal Through (with an $0.1 \mathrm{~mm}$ thick hair in the measuring plane (Stumper, 2003b)) on $S_{11}$ for a high-reflective attenuator is shown for TRL and TRM, whereas the influence is very low for TMSO.

For a low-reflective d.u.t., the influence on $S_{11}$ (and $S_{22}$ ) depends on its attenuation, and the effect for TRL is rather strong, especially for large attenuation (small $S_{12} S_{21}$ ),

$\delta S_{11 \mathrm{~T}}^{\mathrm{TRL}} \approx \frac{\left(L^{2}-S_{12} S_{21}\right)}{\left(1-L^{2}\right)} \cdot \delta T_{11}$,

whereas the effect is small for TRM and for TMSO:

$\delta S_{11 \mathrm{~T}}^{\mathrm{TRM}} \approx-\delta S_{11 \mathrm{~T}}^{\mathrm{TMSO}} \approx-S_{12} S_{21} \cdot \delta T_{11}$.

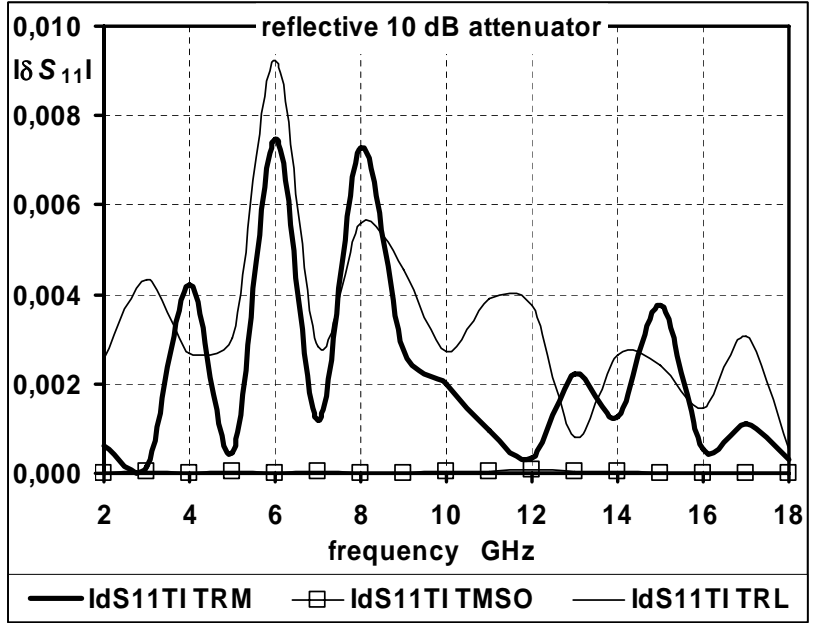

Fig. 8. Comparison of calculated deviations $\left|\delta S_{11 \mathrm{~T}}\right|$ for TRM, TMSO, and TRL calibrations and a high-reflective $10 \mathrm{~dB}$ attenuator, using a non-ideal Through with a $0.1 \mathrm{~mm}$ thick hair in the measuring plane, instead of an ideal Through.

The influence on $S_{12}$ (and $S_{21}$ ) is nearly equal for TRL, TRM, and TMSO (there with opposite sign):

$\delta S_{12 \mathrm{~T}}^{\mathrm{TRL}} / S_{12} \approx \delta S_{12 \mathrm{~T}}^{\mathrm{TRM}} / S_{12} \approx-\delta S_{12 \mathrm{~T}}^{\mathrm{TMSO}} / S_{12} \approx-\delta T_{12}$.

\section{Determination of $g$ for unequal Network reflections}

It can be shown that a determination of the error term $g$ is also possible if the reflection coefficients of the Network $(\mathrm{N})$ are not equal, viz $C_{1} \neq C_{2}$. In this case, an additional connection of the reversed Network to the VNA test ports is necessary. However, such a reversed connection is only possible for equal connector sex at both test ports. For example, this is the case for calibration elements and d.u.t.s in microstrip technique which can be fixed on a test fixture.

For the Network $(\mathrm{N})$ connected directly to the test ports (Index q) and connected reversed (Index r), the S-matrices are

$\overline{\mathbf{N}}_{\mathrm{q}}=\left(\begin{array}{cc}C_{1} & D \\ E & C_{2}\end{array}\right)$ and $\overline{\mathbf{N}}_{\mathrm{r}}=\left(\begin{array}{cc}C_{2} & E \\ D & C_{1}\end{array}\right)$, respectively.

We insert the entries of these two matrices into Eqs. (1) to (4) and obtain for the direct connection:

$a+C_{1} m_{11 \mathrm{Nq}} b g-C_{1} c g+E m_{12 \mathrm{Nq}} d=m_{11 \mathrm{Nq}}$

$D m_{11 \mathrm{Nq}} b g-D c g+C_{2} m_{12 \mathrm{Nq}} d-m_{12 \mathrm{Nq}} g=0$

$C_{1} m_{21 \mathrm{Nq}} b g+E m_{22 \mathrm{Nq}} d-E f=m_{21 \mathrm{Nq}}$

$D m_{21 \mathrm{Nq}} b g+e g+C_{2} m_{22 \mathrm{Nq}} d-C_{2} f-m_{22 \mathrm{Nq}} g=0$,

and for the reverse connection:

$a+C_{2} m_{11 \mathrm{Nr}} b g-C_{2} c g+D m_{12 \mathrm{Nr}} d=m_{11 \mathrm{Nr}}$ 


$$
\begin{aligned}
& E m_{11 \mathrm{Nr}} b g-E c g+C_{1} m_{12 \mathrm{Nr}} d-m_{12 \mathrm{Nr}} g=0 \\
& C_{2} m_{21 \mathrm{Nr}} b g+D m_{22 \mathrm{Nr}} d-D f=m_{21 \mathrm{Nr}} \\
& E m_{21 \mathrm{Nr}} b g+e g+C_{1} m_{22 \mathrm{Nr}} d-C_{1} f-m_{22 \mathrm{Nr}} g=0 .
\end{aligned}
$$

We eliminate $D$ and $E$ from these equations and obtain

$$
\begin{aligned}
& C_{1}=H_{\mathrm{r}} \cdot g / F_{\mathrm{r}} \\
& C_{2}=G_{\mathrm{r}} /\left(I_{\mathrm{r}} \cdot g\right) \\
& C_{1}=G_{\mathrm{q}} /\left(I_{\mathrm{q}} \cdot g\right) \\
& C_{2}=H_{\mathrm{q}} \cdot g / F_{\mathrm{q}}
\end{aligned}
$$

where

$$
\begin{aligned}
& F_{i}=\left(m_{22 \mathrm{Ni}} d-f\right) \cdot\left(m_{11 \mathrm{Ni}} b-c\right)-m_{12 \mathrm{Ni}} m_{21 \mathrm{Ni}} b d \\
& G_{i}=\left(m_{11 \mathrm{Ni}}-a\right) \cdot\left(m_{22 \mathrm{Ni}} d-f\right)-m_{12 \mathrm{Ni}} m_{21 \mathrm{Ni}} d \\
& H_{i}=\left(m_{22 \mathrm{Ni}}-e\right) \cdot\left(m_{11 \mathrm{Ni}} b-c\right)-m_{12 \mathrm{Ni}} m_{21 \mathrm{Ni}} b \\
& I_{i}=\left(m_{11 \mathrm{Ni}} b-c\right) \cdot\left(m_{22 \mathrm{Ni}} d-f\right)-m_{12 \mathrm{Ni}} m_{21 \mathrm{Ni}} b d .
\end{aligned}
$$

The index $i$ is either $q$ or $r$. By equating Eqs. (81) and (83) or Eqs. (82) and (84), we finally obtain:

$g^{2}=g_{\mathrm{qr}}^{2}=F_{\mathrm{q}} G_{\mathrm{r}} /\left(H_{\mathrm{q}} I_{\mathrm{r}}\right)=F_{\mathrm{r}} G_{\mathrm{q}} /\left(H_{\mathrm{r}} I_{\mathrm{q}}\right)$.

The case $D=E=0$ is trivial.

\section{Conclusions}

It has been shown that for the general TAN calibration and derived calibration methods, the error terms and also the deviations of S-parameters of d.u.t.s. due to non-ideal calibration elements can easily be calculated by using the linear-in$\mathrm{T}$ form of the VNA system equations. This is also possible if the Through (T) is a reflectionless network with known transmission parameters. Simple expressions have been obtained for these deviations (sensitivity coefficients). They are suitable for establishing the type-B uncertainty budget for Sparameter measurements, as, in most cases, the deviations from the true S-parameters associated with Through (T), Attenuator (A), and Network (N) can be estimated. Moreover, it is now possible to compare the influence of non-ideal calibration elements for the different calibration methods based on TAN and the 10-term TMSO calibration method. A TAN calibration is also possible in case of unequal reflections of the Network (N).

Acknowledgements. The author would like to thank W. Peinelt, D. Schubert und T. Schrader for carrying out the precise verification measurements.

\section{References}

Engen, G. F. and Hoer, C. A.: Thru-Reflect-Line: An Improved Technique for Calibrating the Dual Six-port Automatic Network Analyzer, IEEE Trans. Microwave Theory Tech., 27, 987-993, 1979.

Eul, H.-J. and Schiek, B.: A Generalized Theory and New Calibration Procedures for Network Analyzer Self-Calibration, IEEE Trans. Microwave Theory Tech., 39, 724-731, 1991.

Gronau, G.: Höchstfrequenztechnik. Berlin, Springer, Ch. 8, 389398, 2001.

Rytting, D.: Network Analyzer Error Models and Calibration Methods, ARFTG/NIST Short Course on RF Measurements for a Wireless World, San Diego, CA, Nov. 29-30, 2001.

Schiek, B.: Grundlagen der Hochfrequenz-Messtechnik. Berlin, Springer, Ch. 4.4, p. 159, 1999.

Stumper, U.: Influence of TMSO Calibration Standards Uncertainties on VNA S-Parameter Measurements, IEEE Trans. Instrum. Meas., 52, 311-315, 2003a.

Stumper, U.: Uncertainty of VNA S-Parameter Measurement due to Non-Ideal TMSO or LMSO Calibration Standards, Advances in Radio Science (Kleinheubacher Berichte), [Online], 1, 1-8, available: http://www.copernicus.org/URSI/ars/1/1.pdf, 2003b.

Stumper, U.: Uncertainty of VNA S-Parameter Measurement due to Non-Ideal TRL Calibration Items, IEEE Trans. Instrum. Meas., 55, 676-679, 2005a.

Stumper, U.: Fehlerfortpflanzung bei der LRL- oder TRL-Kalibrierung eines vektoriellen VierstellenNetzwerkanalysators, in: Neue Kalibrierverfahren im Niederund Hochfrequenzbereich - Vorträge des 206. PTB-Seminars, edited by: Bachmair, H., PTB-Bericht E-89, Braunschweig, May 2005b.

Stumper, U.: Influence of Non-ideal LRL or TRL Calibration Elements on VNA S-Parameter Measurements, Advances in Radio Science (Kleinheubacher Berichte), [Online], 3, 51-58, available: http://www.copernicus.org/URSI/ars/3/ars-3-51.pdf, 2005c. 